\title{
Demonstration of a stable ultrafast laser based on a nonlinear microcavity
}

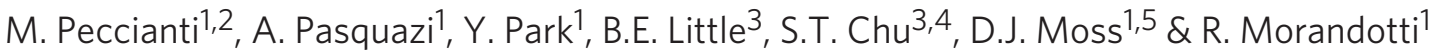

Ultrashort pulsed lasers, operating through the phenomenon of mode-locking, have had a significant role in many facets of our society for 50 years, for example, in the way we exchange information, measure and diagnose diseases, process materials, and in many other applications. Recently, high-quality resonators have been exploited to demonstrate optical combs. The ability to phase-lock their modes would allow mode-locked lasers to benefit from their high optical spectral quality, helping to realize novel sources such as precision optical clocks for applications in metrology, telecommunication, microchip-computing, and many other areas. Here we demonstrate the first mode-locked laser based on a microcavity resonator. It operates via a new mode-locking method, which we term filter-driven four-wave mixing, and is based on a CMOS-compatible high quality factor microring resonator. It achieves stable self-starting oscillation with negligible amplitude noise at ultrahigh repetition rates, and spectral linewidths well below $130 \mathrm{kHz}$.

\footnotetext{
1 INRS-EMT, 1650 Blvd. Lionel Boulet, Varennes Québec, J3X 1S2 Canada. ${ }^{2}$ IPCF-CNR, UOS Roma and ISC-CNR UOS Montelibretti, Via dei Taurini 19, 00185 Roma, Italy. ${ }^{3}$ Infinera Ltd, 169 Java Drive, Sunnyvale, California 94089, USA. 4 Department of Physics and Materials Science, City University of Hong Kong. ${ }^{5}$ CUDOS and the Institute of Photonics and Optical Science (IPOS), School of Physics, University of Sydney, Sydney, New South Wales 2006 , Australia. Correspondence and requests for materials should be addressed to A.P. (email: alessia.pasquazi@gmail.com).
} 
$\mathrm{P}$ assively mode-locked lasers have generated the shortest optical pulses to date ${ }^{1}$. Self-supporting ultrashort optical pulses can be produced naturally in these lasers ${ }^{1,2}$ by the complex nonlinear interactions between chromatic dispersion, the Kerr nonlinearity and saturable gain ${ }^{3,4}$. There is considerable interest in achieving both very high and flexible repetition rates ${ }^{5,6}$ at frequencies well beyond the range of active mode-locking, where the use of electronics poses intrinsic limitations. Many different approaches have been proposed to achieve this, ranging from very short laser cavities with large mode frequency spacings (for example, large free spectral range $(\mathrm{FSR})^{1,7,8}$, where a very high repetition rate is achieved by simply reducing the pulse round-trip time, at the expense of line quality), to schemes where multiple pulses are produced in each round trip ${ }^{3,9,10}$, applied primarily to fibre lasers. A pioneering approach to the latter was introduced in 1997 by Yoshida et al. ${ }^{10}$, where a Fabry-Pérot filter inserted in the main cavity suppresses all but a few modes that are periodically spaced, leading to a train of pulses with a controlled repetition rate. This approach was subsequently reinterpreted in terms of the dissipative fourwave-mixing (FWM) paradigm ${ }^{11,12}$, and since then, high repetitionrate pulse trains have been demonstrated by adopting variations of this method ${ }^{12-15}$. Although, in principle, dissipative FWM schemes allow for transform-limited pulses to be generated at repetition rates not limited by the main laser cavity, their stability still remains a severe issue-a common problem ${ }^{1}$ when multiple pulses circulate in a cavity. This is a consequence of the fact that the gain and nonlinearity required to sustain proper lasing action necessitate the use of very long fibre cavity lengths. This, in turn, produces smaller cavity mode frequency spacings of (typically) a few megahertz or less, which allows many modes to oscillate within the Fabry-Pérot filter bandwidth. Because these modes have random phases, their beating results in severe low-frequency noise that produces extremely unstable operation ${ }^{14}$.

In this paper, we report the first mode-locked laser based on a nonlinear monolithic high-Q (quality) factor resonator. Our laser achieves extremely stable operation at high repetition rates while maintaining very narrow linewidths, thus leading to a high-quality pulsed emission. The key point is that this resonator is not simply used as a filter but acts as the nonlinear element as well ${ }^{16}$, similar in spirit to optically pumped multiple wavelength oscillators based on high Q-factor resonators ${ }^{16-22}$. Because of this twofold central role of the nonlinear filter, we term this new mode-locking scheme filter-driven four-wave mixing (FD-FWM). It operates in a way that is in stark contrast to traditional dissipative FWM schemes, where the nonlinear interaction occurs in the fibre and is then filtered separately by a linear Fabry-Pérot filter.

\section{Results}

General features of filter-driven four-wave mixing laser. Our method has two immediate advantages. First, it is intrinsically more efficient; in the alternate approach, the nonlinear mixing is usually performed right after the amplification stage, in the point of the loop where the pulse energy is maximum. The linear filtering follows the nonlinear step, cutting part of the spectral broadening. In our design, the filtering cannot be considered to be separated from the nonlinear process. As some frequency components simply do not survive in the filter owing to destructive interference, they are not able to seed the energy transfer process towards forbidden bands in the spectrum, consequently reducing the general power loss. Second, it drastically reduces the main cavity length, which substantially increases the main cavity mode frequency spacing, thus dramatically increasing the laser stability, as, ideally, only one cavity mode is now allowed to oscillate within the ring resonance. The combination of these factors enables our scheme to achieve stable operation at high repetition rates over a large range of operating conditions, without any extra stabilization system. In addition, our FD-FWM scheme can intrinsically produce much narrower linewidths than ultrashort cavity mode-locked lasers (that oscillate at similar repetition rates), because the long main cavity results in a much smaller SchawlowTownes phase noise limit ${ }^{23,24}$.

Thermal stability of filter-driven four-wave mixing scheme. A further key advantage of our approach lies in the fact that the stable lasing regime is highly robust to external (for example, thermal) perturbations, that are a well-known problem in resonator-based optical parametric oscillators (OPOs) $)^{17,22}$. In OPOs, temperature variations due to the circulating power naturally detune filter resonances with respect to an external pumping source. Although stabilization mechanisms such as thermal locking have been exploited to solve this problem, they are ineffective against slow temperature drifts, a fact which often leads to the situation where the OPO shuts down ${ }^{25}$. Our approach does not suffer from this problem as all the oscillating lines are actually modes of the main cavity that lase within the resonator resonances. As the nonlinear resonator is part of the main cavity, the changes in the resonator optical path induced by heating also contribute to a change in the main cavity length, which in turn moderates the frequency misalignment between ring and main cavity modes. Moreover, the stable oscillation is sustained primarily by external gain and it is obtained at resonator input power levels in the $10 \mathrm{~mW}$ range, about an order of magnitude lower than the values normally used to pump OPOs. The ring resonator heating issues are therefore reduced. However, we found that the central lasing frequency was dependent on the energy coupled in the ring, which yielded a variation of the central frequency over the power at the input of the ring of approximately $0.67 \mathrm{pm} \mathrm{mW}^{-1}$.

Laser set-up and operation. The key component of the laser, the ring resonator, shown in Fig. $1 \mathrm{a}-\mathrm{d}$, is an integrated microring resonator with a $Q$ factor of 1.2 million (refs 21, 26) (estimated from a measured linewidth of $160 \mathrm{MHz}$ ), fabricated in a CMOS-compatible chip platform ${ }^{27,28}$ based on a high-index, doped-silica glass waveguide. The experimental set-up is shown in Fig. $1 \mathrm{~b}$ (see Methods for details). The microring resonator is simply embedded in a standard erbium doped-fibre loop cavity (acting as the gain medium) containing a passband filter with a bandwidth large enough to pass all of the oscillating lines, with the main purpose of controlling the central wavelength $\lambda_{0}$. A delay line is employed to control the phase of the main cavity modes with respect to the ring modes.

To investigate the dependence of the laser performance on the main cavity length, we conducted experiments with two lasers, one based on a short-length erbium-doped fibre amplifier (EDFA) and the other based on a long high-power erbium-ytterbium fibre amplifier (EYDFA), the latter having a similar operating regime to dissipative FWM lasers, where long fibre-based cavities are necessary to assure the Kerr nonlinearity required for mode-locked laser operation. The two configurations therefore had significantly different main cavity lengths ( 3 and $33 \mathrm{~m}$ ), that is, different FSRs (68.5 and $6 \mathrm{MHz}$, respectively) as well as different saturation powers.

Figure 2 shows the experimental optical spectra of the pulsed output (Fig. 2a,c) along with the temporal traces obtained by a second-order noncollinear autocorrelator (Fig. 2b,d) for the two systems (EYDFA in Fig. 2a,b and EDFA in Fig. 2c,d) at four input powers to the ring resonator. The pulses visible in the autocorrelation trains have a temporal duration that decreases noticeably as the input power increases, as expected for a typical passive modelocking scheme.

From these plots, it would appear that the laser based on the high-power EYDFA had superior overall performance because its pulsewidth was considerably shorter. However, this ignores the fact that the autocorrelation measurements average over any long-time scale fluctuations of the laser, without requiring a stable pulsed output. The key issue of laser stability or line coherence is better illustrated 
a
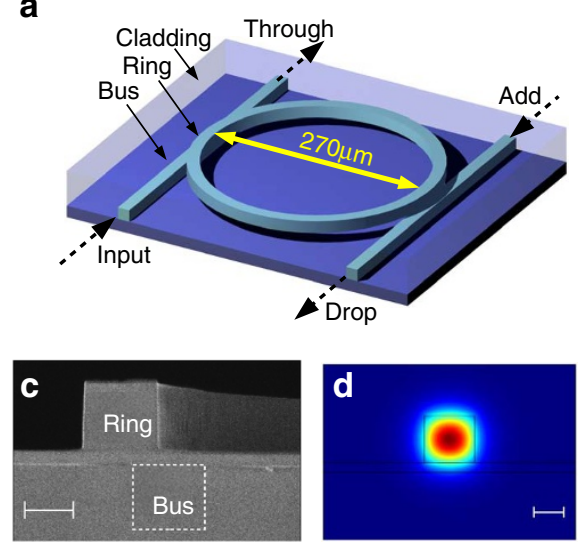

b

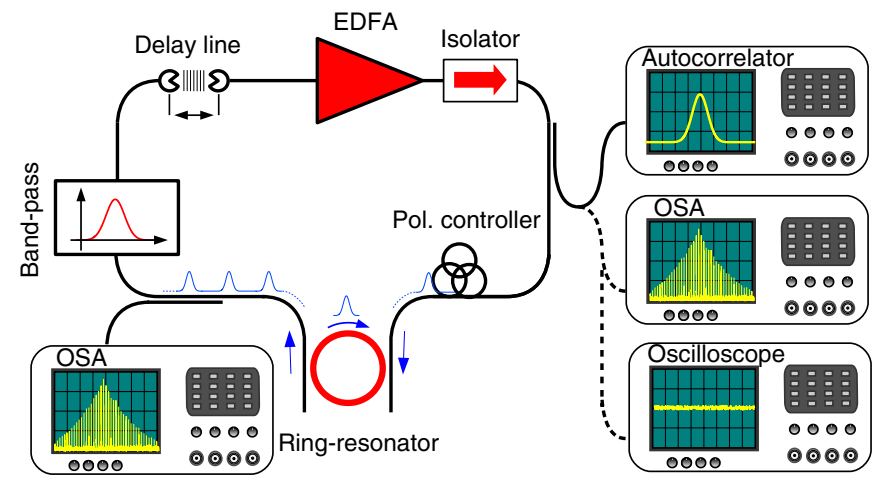

Figure 1 | Filter-driven four-wave mixing design. (a) Schematic of the central component - a monolithically integrated 4-port high- $Q(Q=1.2$ million) microring resonator (fibre pigtails not shown) (b) High repetition rate laser based on filter-driven FWM: the microring resonator in (a) is embedded in a loop cavity containing a gain fibre (EDFA), band-pass filter with the main purpose of controlling the central wavelength $\lambda_{0}$, a delay line to control the phase of the main cavity modes with respect to the ring modes, an isolator to force the pulse-circulating direction, and a polarization controller to act on the pulse polarization, as the ring resonator is birefringent. The waveform at the output of the amplifier is monitored with an autocorrelator to measure the pulse duration, and an optical spectrum analyser (OSA) and a photodiode connected to an oscilloscope to respectively measure the optical and radio frequency spectrum of the waveform. An OSA is also employed to monitor the optical spectrum at the output of the ring resonator. (c) SEM picture of the ring cross-section before depositing the upper cladding of $\mathrm{SiO}_{2}$. The waveguide core is made of high index $(n=1.7)$ doped silica glass. The scale bar represents $1 \mu \mathrm{m}$. (d) Electric field modal distribution for a TM polarized beam calculated through vectorial mode-solving. The scale bar represents $1 \mu \mathrm{m}$.

by a comparison between the experimental autocorrelation traces with the calculated traces (Fig. 2b,d, green plots) for a fully stable and coherent system possessing the optical spectra in Fig. 2a,c. Whereas a perfect match is found for the short-length EDFA case, the long cavity design shows a considerably higher background, thus clearly distinguishing unstable from stable laser operation ${ }^{22}$.

By changing the amplifier driving current, we observed a minimum lasing threshold of 500 and $650 \mathrm{~mA}$ for the unstable and stable designs, respectively (Fig. 2e,f) that corresponded to a gain of $\sim 20 \mathrm{~dB}$ for both the cases. For the stable case, the input power in the ring versus the driving current is plotted in Fig. $2 \mathrm{f}$. To preserve the stable operation, the delay line was adapted at the mode-locking threshold. This yielded a slight change in total losses that induced a kink in the plot around a current of $850 \mathrm{~mA}$. The maximum input power in the ring was $15.4 \mathrm{~mW}$ for the stable case, limited by the performance of the EDFA.

\section{Discussion}

To better quantify the pulse-to-pulse amplitude stability of the two lasers we recorded the electrical radio-frequency (RF) spectrum of the envelope signal, collected at the output, using a fast photodetector. Unstable oscillation (in the pulse amplitude) was always observed for the long cavity design for the EYDFA (Fig. 3a,b) for both continuous wave $(\mathrm{CW})$ and pulsed regimes, owing to the presence of a large number of cavity modes oscillating in the ring resonance. In complete contrast to this, the short-cavity configuration for the EDFA (Fig. 3c-f) could easily be stabilized to give the very clean result of Fig. $3 \mathrm{e}, \mathrm{f}$, by simply adjusting the main cavity length to centre an, ideally, single cavity mode with respect to the ring resonance, thereby completely eliminating any main cavity low-frequency beating. Several stable oscillation conditions were found through tuning the delay by over $2 \mathrm{~cm}$. For the same gain, the optical bandwidth (insets in Fig. 3c,d) for unstable laser operation was wider (that is, leading to shorter output pulses) because the instability resulted in a strong amplitude modulation of the optical pulse train in the main cavity, thus increasing the statistical peak power and enhancing the nonlinear interactions. This is a common occurrence in modelocked lasers ${ }^{29}$.
Although we expect that significant external temperature changes would eventually detune the external cavity length, once the thermal stability was reached, standard environmental conditioning $\left(21^{\circ} \mathrm{C}\right)$ was sufficient to maintain perfectly stable operation within the time-framework of our experimental sessions (several hours), highlighting the robustness of the stability condition.

We confirmed, by numerical simulations (Methods), the dependence of the stability on the relative phase between the main cavity modes and the ring resonator modes (Fig. 4). We found that instability could indeed be either induced or suppressed simply by optimizing the phase of the main cavity modes, that is, the spectral position of the main cavity modes relative to the ring resonances.

We concluded our experiments by characterizing the phase noise $\mathrm{e}^{7,24,30-32}$. We found that all of the emitted lines had a bandwidth well below $130 \mathrm{kHz}$ (full-width at half-maximum (FWHM)). This bandwidth has been found to be dramatically increased by the contribution of the environmental acoustic noise that we could minimize but not fully eliminate. Following the approach of ref. 24 , we discriminated the linewidth associated with the inherent bandwidth-quantum-limit, estimated to be below $13 \mathrm{kHz}$, which suggests that, ultimately, proper packaging and acoustic isolation would reduce the optical spectral linewidth even further. We also investigated the source timing jitter, that is, the stochastic deviation of the temporal pulse position in the train. Following refs. 7,30-32, we estimate from the phase noise that the jitter contribution to the RF spectral lines is $<10 \mathrm{KHz}$. This is a remarkable result for a $200 \mathrm{GHz}$ mode-locked source that does not use any auxiliary stabilization mechanisms.

Our ultimate objective is a versatile, fully monolithically integrated laser source. Because waveguide amplifiers have been demonstrated in silica glass platforms, we do not envisage any fundamental issues preventing the full integration of our scheme. This proof-of-concept device represents a key step in realizing a fully integrated, stable, high-performance, laser source operating at flexible and very high repetition rates.

In summary, we propose and demonstrate a novel mode-locked laser based on a monolithic high-Q resonator, capable of generating 
a
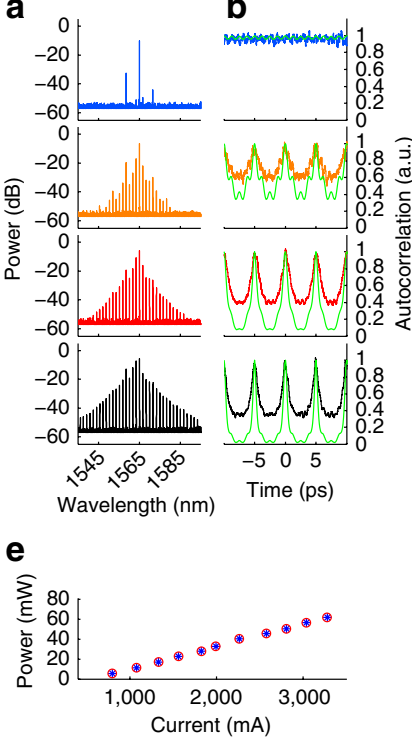

c

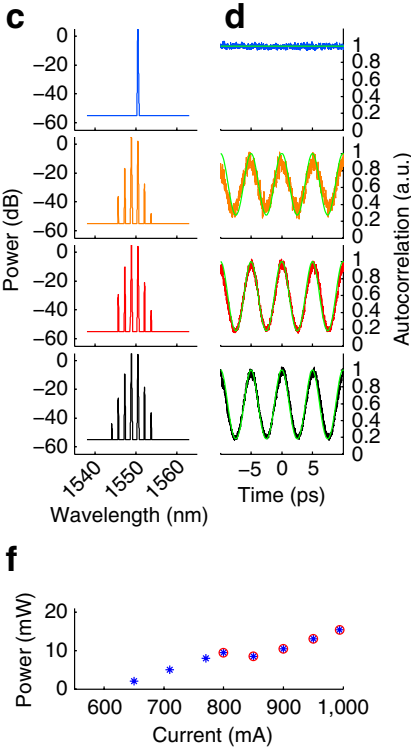

Figure 2 | Experimental optical spectra and autocorrelation traces of the laser output for different FSRs of the main cavity. $(\mathbf{a}, \mathbf{b})$ Long cavity (FSR $=6.0 \mathrm{MHz}$ ) laser emission for increasing (top to bottom) pump powers, achieved through the use of a long EYDFA, for 5.5 (blue), 28 (orange), 40 (red) and $68 \mathrm{~mW}$ (black) average ring input powers. (a) shows the optical power spectrum measured with the OSA and (b) gives the temporal waveform of the laser measured with the autocorrelator. Autocorrelation traces calculated starting from the experimental optical spectra for a fully coherent and transform-limited system are shown in green. These profiles are calculated by considering each line of the experimental optical spectra as being perfectly monochromatic and inphase with the others, yielding an output pulse with a FWHM of $730 \mathrm{fs}$ (duty cycle $\rho=0.15$ ) for the highest excitation power condition. The measured autocorrelation shows a considerably higher background (the peak-to-background ratio is 2.5:1 for the $68 \mathrm{~mW}$ case) than the expected autocorrelation (50:1). (c, d) Short-cavity laser emission using a shortlength, low-power EDFA. (c) shows the optical power spectrum and (d) is the temporal waveform of the laser output. Average powers in the ring were as low as 8.0 (blue), 10.4 (orange), 13.0 (red) and $15.4 \mathrm{~mW}$ (black), with the main cavity FSR $=68.5 \mathrm{MHz}$. Because of the lower cavity energy achievable using the short-length EDFA, we show here a maximum spectral bandwidth limited to seven or eight lasing modes associated with the $200 \mathrm{GHz}$ FSR of the ring resonator. The autocorrelation traces expected from the optical spectra for a fully coherent transform-limited system, shown in green, perfectly match the measured traces, corresponding to a transform-limited pulse width (FWHM) of 2.3 ps (duty cycle $\rho=0.46$ ), with a peak to background ratio of 5:1 for the $15.4 \mathrm{~mW}$ case. In all cases, the oscillation condition was self-starting and did not critically depend on the initial conditions. (e,f) Average input power in the ring versus the driving current for the EYDFA and the EDFA, respectively. Red circles indicate multiwavelength oscillation. In the EDFA case, the delay line was adjusted to keep the system in stable operation for driving currents $>850 \mathrm{~mA}$.

both picosecond and sub-picosecond transform-limited pulses at a repetition rate of $200.8 \mathrm{GHz}$ and beyond. Our device operates via a new mechanism that enables stable mode-locked lasing with negligible amplitude noise, and extrinsically limited phase noise, not constrained by the repetition rate. We believe this work represents a key milestone in the generation of ultra-stable, high repetition rate, optical pulse sources, particularly because of its CMOS-compatible monolithic platform.
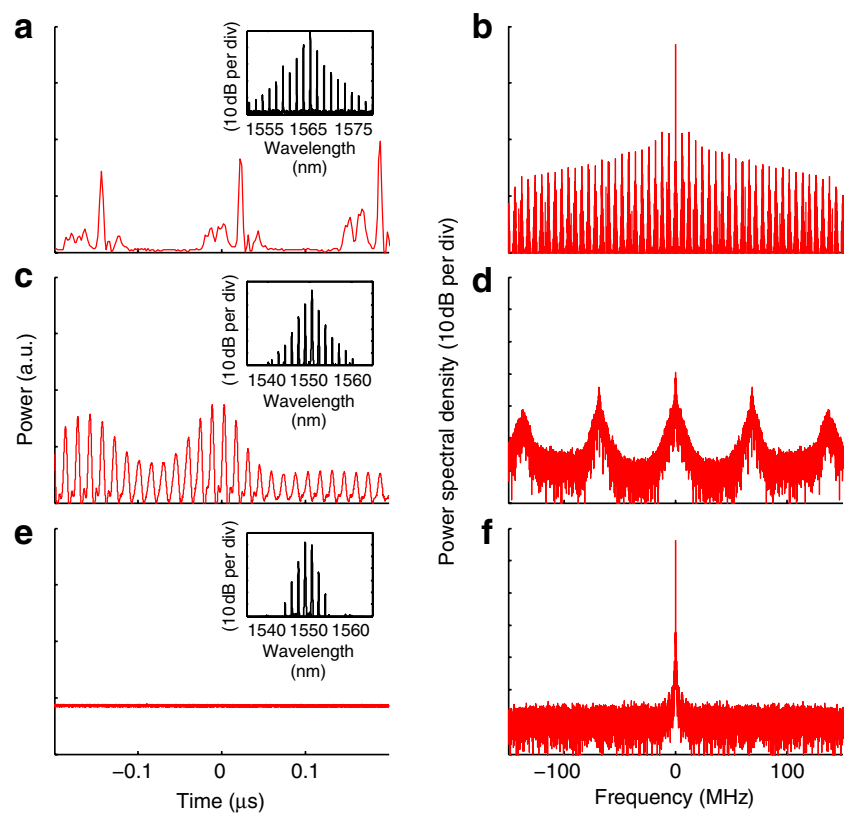

Figure $\mathbf{3}$ | Laser RF noise. (a,b) Unstable oscillation condition for a main cavity $F S R=6 \mathrm{MHz}$, obtained using the EYDFA (average ring input power $68 \mathrm{~mW}$ ). The inset in (a) shows the optical spectrum, as measured with the OSA. In (a), the RF signal clearly shows an irregular pulsation. This is reflected in the appearance of harmonics in the RF spectrum (b) at $6 \mathrm{MHz}$ intervals. The limited bandwidth of the RF spectrum is consistent with the linewidth $(160 \mathrm{MHz})$ of each ring resonator line. (c,d) Unstable oscillation condition for FSR $=68.5 \mathrm{MHz}$ obtained using the EDFA (multiple cavity modes oscillating per filter resonance). In this configuration, the long timescale output shows complex temporal behaviour, with the RF spectrum containing peaks corresponding to $68.5 \mathrm{MHz}$-spaced harmonics. The inset in (c) shows the optical spectrum, as measured with the OSA. (e,f) Stable oscillation condition for $\mathrm{FSR}=67 \mathrm{MHz}$, corresponding to the case of one cavity mode oscillating per filter resonance. The average power in the ring was $15.4 \mathrm{~mW}$. The inset in (e) shows the optical spectrum. This regime is obtained by adjusting the phase of the cavity modes relative to the ring resonator modes via a free space delay line.

\section{Methods}

Simulations. The numerical simulations were performed by solving the coupled equations of the field evolution $f(z, t)$ in the amplifying fibre and of the field $a(z, t)$ evolution in the nonlinear ring

$$
\begin{aligned}
& \partial_{z} f(z, t)+\frac{n_{\mathrm{F}}}{c} \partial_{t} f(z, t)+i \frac{\beta_{2 \mathrm{~F}}}{2} \partial_{t t} f(z, t)=g(f)\left(1-\frac{\partial_{t t}}{\Omega}\right) f(z, t)-\alpha f(z, t) \\
& \partial_{z} a(z, t)+\frac{n_{\mathrm{R}}}{c} \partial_{t} a(z, t)+i \frac{\beta_{2 \mathrm{R}}}{2} \partial_{t t} a(z, t)+i \gamma|a(z, t)|^{2} a(z, t)=0
\end{aligned}
$$

Here $c$ is the speed of light, $n_{\mathrm{F},} n_{\mathrm{R}}$ and $\beta_{2 \mathrm{~F}} \beta_{2 \mathrm{R}}$ are the group indices and secondorder dispersion in the fibre $(\mathrm{F})$ and in the ring $(\mathrm{R})$, respectively. Also, $\gamma$ is the Kerr nonlinear coefficient in the ring, whereas for the fibre, $\alpha$ is the linear absorption and $g(f)$ is the saturable gain expressed as

$$
g(f)=G_{0}\left[\exp \left(-\int_{0}^{L}|f(z, t)|^{2} \frac{d z}{P_{0} L}\right)\right]
$$

with $G_{0}$ and $P_{0}$ representing the fibre low-signal gain and the amplifier saturation power, which controls the energy in the laser cavity, and $L$ is the main cavity length. We also note that $\Omega$ regulates the fibre gain bandwidth. The equations are solved in time with a pseudo-spectral method and are coupled at the ring ports 
according to the relation

$$
\left(\begin{array}{l}
a_{\text {out }} \\
f_{\text {out }}
\end{array}\right)=\left(\begin{array}{cc}
t & r \\
-r & t
\end{array}\right)\left(\begin{array}{l}
a_{\text {in }} e^{i \Phi_{\mathrm{RC}}} \\
f_{\text {in }} e^{i \Phi_{\mathrm{MC}}}
\end{array}\right)
$$

where $t$ and $r$ are the transmission and reflection coefficients that regulate the ring bandwidth, $\Phi_{\mathrm{MC}}$ dictates the position of the main cavity modes with respect to the ring modes, and $\Phi_{\mathrm{RC}}$ fixes the position of the ring modes with respect to the centre of the gain bandwidth.

The simulations were performed for a $200 \mathrm{GHz}$ repetition rate system, starting from noise and letting the system reach the stationary state. The main cavity is shorter when compared with the experiment, to fit the problem within the 32 Gbyte memory of our multiprocessor system. The operation is investigated for a ring line $\mathrm{FWHM}_{\mathrm{RC}}=30 \mathrm{GHz}$ and a main cavity $\mathrm{FSR}_{\mathrm{MC}}=12 \mathrm{GHz}$. The ratio $\mathrm{FWHM}_{\mathrm{RC}} / \mathrm{FSR}_{\mathrm{MC}}=2.5$ has been chosen to match the experimental conditions. The dispersion is scaled accordingly to obtain a total dispersion corresponding to the experiments.

In Fig. 4a-f, the simulation results (output spectrum and amplitude) are plotted for growing amplifier saturation energy (from blue to black), for $\Phi_{\mathrm{MC}}=0$ (Fig. $4 \mathrm{a}-\mathrm{c}$ ) and $\Phi_{\mathrm{MC}}=0.875 \pi$ (Fig. $4 \mathrm{~d}-\mathrm{f}$ ). The cavity energy affects the stability as it regulates the number of main cavity modes that oscillate (Fig. 4b,e). Configurations with lower energy and modes centred exactly on a ring resonator resonance $\left(\Phi_{\mathrm{MC}}=0 \mathrm{rad}\right)$ are typically stable. The parameter used in the experiments to distinguish the stable and unstable regimes is the bandwidth of the RF spectrum. In the numerics, it corresponds to the low-frequency components $(<100 \mathrm{GHz}$ in the simulations) of the spectrum of the temporal power waveform $\left|f_{\text {out }}(t)\right|^{2}$. In Fig. $4 \mathrm{~g}$ the FWHM of the RF spectrum versus $\Phi_{\mathrm{MC}}$ is shown for different amplifier saturation powers. This quantity is expressed in units of main cavity modes (operatively, the FWHM is divided by the FSR of the main cavity), that is, $\mathrm{FWHM}_{\mathrm{RF}}=1 \mathrm{MC}_{\text {modes }}$ represents a bandwidth as large as the FSR, and it corresponds to a moderate unstable behaviour, for example, the red line in Fig. $4 \mathrm{a}-\mathrm{c}$. As it is visible, in Fig. $4 \mathrm{~g}$, the stable operating range in terms of phase delay $\Phi_{\mathrm{MC}}$ shrinks with the cavity energy.

Device. The waveguides used to fabricate the high-Q ring resonators in our experiment possess a high effective nonlinearity $\left(220 \mathrm{~W}^{-1} \mathrm{~km}^{-1}\right)$ due to a combination of tight mode confinement and high intrinsic Kerr coefficient $\left(n_{2}\right.$ is five times that of silica glass). The dispersion is optimized for FWM in the C-band as it is small and anomalous $\left(\sim-10 \mathrm{ps}^{2} \mathrm{~m}^{-1}\right)$ near $\lambda=1550 \mathrm{~nm}$ (ref. 26), guaranteeing a large FWM gain when pumped in the C-Band. Most significantly, the waveguides exhibit very low linear $\left(<0.04 \mathrm{~dB} \mathrm{~cm}^{-1}\right)$ and negligible nonlinear optical loss (for example, two photon absorption) up to $25 \mathrm{GW} \mathrm{cm}^{-2}$. All of these factors make this platform particularly attractive for low-power nonlinear optics in the C-band ${ }^{21,26-28}$. The input and output bus waveguides of the resonator are pigtailed to standard single mode fibre (SMF) using integrated mode converters and a V-groove technology, resulting in coupling losses of $<1.5 \mathrm{~dB}$ per facet.

Set-up and experiments. The microring resonator is embedded in a larger fibre loop cavity, containing an optical amplifier to provide gain, a Faraday isolator, a polarization controller and a free space delay line. A passband filter, with a bandwidth large enough to pass all of the oscillating lines, is used to control the central wavelength $\lambda_{0}$. The optical amplifiers used to perform the experiments reported here were either a short-length EDFA, from PriTel, or a more conventional (longer length) high-power erbium-ytterbium doped fibre amplifier (EYDFA). The EYDFA had a maximum saturation output power of $\sim 3 \mathrm{~W}$, and produced a main cavity FSR of $6.0 \mathrm{MHz}$, corresponding to a main cavity fibre length of $33 \mathrm{~m}$, whereas the shortlength EDFA had a saturation output power of $\sim 35 \mathrm{~mW}$ and resulted in a cavity length below $3 \mathrm{~m}$, corresponding to a very large main cavity FSR of $68.5 \mathrm{MHz}$. For the EYDFA-based laser, the bandpass filter in the fibre loop used to control the central oscillating wavelength was a $15-\mathrm{nm}$ wide filter with $\sim 4 \mathrm{~dB}$ loss. The filter imposes a gain difference of $\sim 0.5 \mathrm{~dB}$ between the central and the first side modes. For the short-length laser, a 5-nm wide filter was used instead, characterized by a loss of $1.5 \mathrm{~dB}$. We verified that the use of different filters for each laser had no impact on the performances in terms of overall stability or linewidth-the filter bandwidth was chosen to optimize the performance of each laser. For the EYDFA-based laser, much higher pump powers were available, so decreasing the loss was not as important as providing a wide-enough bandwidth to achieve the shortest possible pulsewidth. For the short laser, a limited gain was available and hence the low-loss, narrow bandwidth filter was chosen; also because the overall output bandwidth was much smaller than the EYDFA laser owing to the lower available cavity energy. The central lasing wavelengths of the two lasers were adjusted to obtain the maximum achievable bandwidth. The different optimal operating wavelength is mainly related to the different main cavity dispersion (anomalous in both cases) and different amplifier gain profiles.

Amplitude noise characterization. The laser amplitude noise was determined by measuring the electrical RF spectrum of the laser using a fast photodetector connected to an amplifier (bandwidth, $200 \mathrm{MHz}$ ).
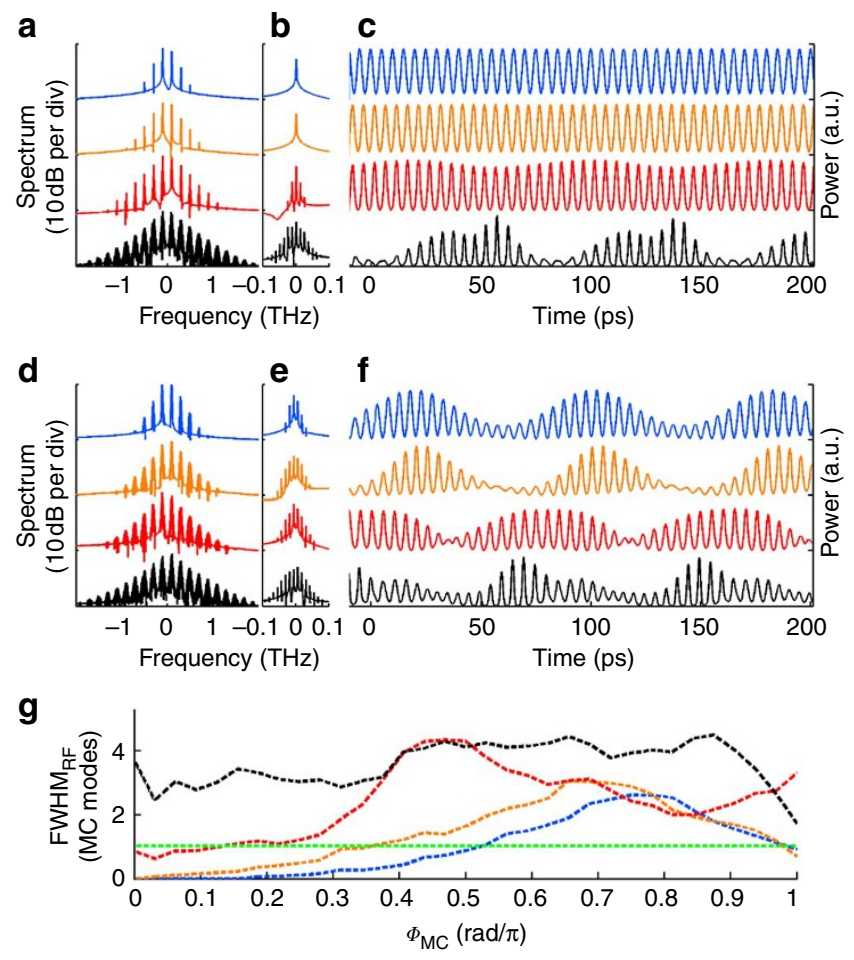

Figure 4 | Theoretical dependence of laser stability on amplifier saturation energy and phase $\Phi_{\mathbf{M C}}$ of the main cavity modes. In all the plots, $\Phi_{\mathrm{RC}}=\pi$ (even number of oscillating ring resonances). The blue, orange, red and black plots are for growing cavity energy, respectively, 1, 2, 3 and 4 times the amplifier saturation energy associated with the blue line. (a-c) Lasing condition for $\Phi_{M C}=0$. (a,b) Optical spectrum. (c) Temporal evolution, showing modulated peaks in the unstable cases. (d-f) Lasing condition for $\Phi_{M C}=0.875 \pi$. The plots (b,e) show a zoom on the central ring lines in $(\mathbf{a}, \mathbf{d})$, where the main cavity modes are clearly distinct (corresponding to only one line in the stable case). For larger excitations, the number of oscillating main cavity modes increases. (g) RF bandwidth (FWHM) as a function of the main cavity modes phase $\Phi_{M C}$, expressed in units of number of main cavity modes (for example, as the ratio between the RF bandwidth and the main cavity FSR).

For the stable case (Fig. 3e), the RF signal exhibited a dominant component centred at the zero frequency (namely 'direct current' (DC) component), with a bandwidth $<0.25 \mathrm{~Hz}$ (the resolution of our measurement system) as well as an outof-band noise $55 \mathrm{~dB}$ lower than the DC peak. We estimate the ratio between the power of the DC component and spectral noise (within the $200 \mathrm{MHz}$ bandwidth) to be $>41 \mathrm{~dB}$, again limited by the sensitivity of the measurement, which in our case is dictated by detection and sampling noise. For the unstable case (Fig. 3a), the RF bandwidth is very large and the DC component brings actually a quite weak spectral contribution, the ratio always being $\ll 0 \mathrm{~dB}$. We estimate this to be $\sim-13 \mathrm{~dB}$ for the $6 \mathrm{MHz}$ FSR main cavity.

Phase-noise characterization. To characterize the phase noise, we measured the linewidth of the oscillating lines by first selecting each of them with a narrowband filter and then by analysing the output using a re-circulating delayed self-heterodyne interferometer (RDSHI) with a delay line of $5.8 \mathrm{~km}$, and a modulation frequency of $6.5 \mathrm{MHz}$ (refs 7,24,30-32). As reported in Fig. 5, we found that all of the observable emitted lines had a bandwidth well below $130 \mathrm{kHz}$ (FWHM), only limited by the environmental acoustic noise, a factor that we could minimize but not completely eliminate. This is consistent with our best-fit estimation of the intrinsic Lorentzian contribution to the linewidth being $<13 \mathrm{kHz}$, that is, the quantum limit for the linewidth ${ }^{24}$, and implies that, ultimately, proper packaging and acoustic isolation would significantly reduce the RF spectral linewidths. Notice that the widths of the emission lines of the unstable design were exactly matching the ring bandwidth, as also confirmed by the output amplitude spectrum.

Although it is not possible to obtain the pulse-jitter amplitude directly from the RF spectrum ${ }^{31}$ owing to the extremely high repetition rate, the pulse jitter can be estimated from the the optical spectral broadening $\Delta w_{n}$ of the RF laser lines $7,30-32$. Under the assumption that the phase and time jitter noises are white 

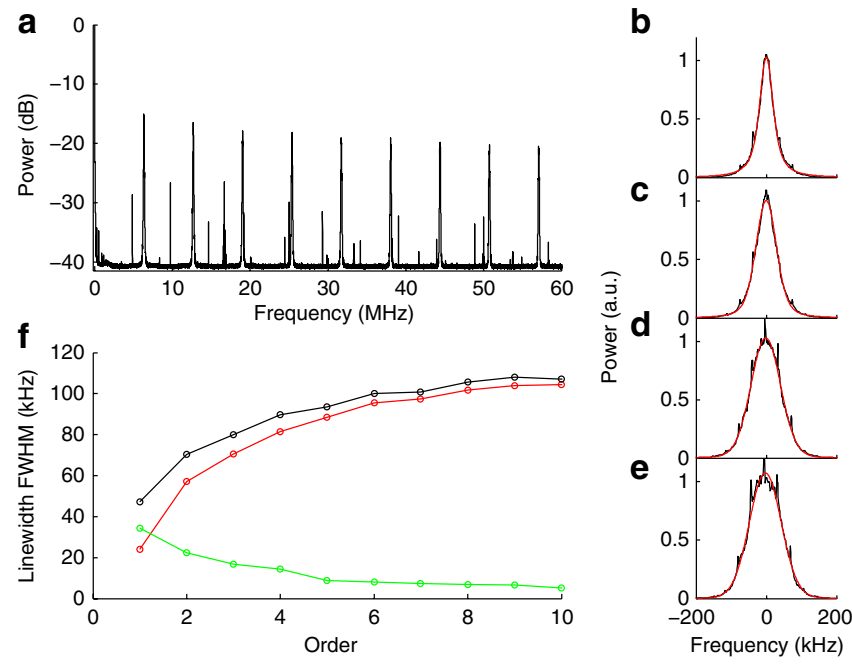

Figure 5 | Phase noise measurement performed using a re-circulating delayed self-heterodyne interferometer. Measurements were performed with a delay line of $5.8 \mathrm{~km}$, and a modulation frequency of $6.5 \mathrm{MHz}$ (refs 7,24,30-32). The set-up measurement is described in ref. 30. (a) Broadband view of the RDSHI photocurrent power spectrum. The recirculating beat notes are separated by the modulation frequency of $6.5 \mathrm{MHz}$. (b-e) RDSHI photocurrent power spectra of the first, second, sixth and tenth beat notes (black), respectively, along with a best fit to the Voigt function (red). The Voigt function is defined as the convolution of a Gaussian and a Lorentzian function (representing the extrinsic and intrinsic contribution to the noise, respectively), as in ref. 24. (f) Linewidth FWHM (black dots), Lorentzian (green dots) and Gaussian (red dots) contributions of the Voigt best fit function versus the order of the beat note. From this measurement, it is clear that the Lorentzian contribution (that is, the quantum limit for the linewidth) saturates to a value well below $13 \mathrm{kHz}$, whereas the Gaussian contribution is dominant for the largest order, indicating that the noise is dominated by extrinsic/environmental contributions.

and uncorrelated ${ }^{32}$ the spectral broadening $\Delta w_{\text {jitter }}$ due to the timing jitter is known to be a parabolic coefficient of $\Delta w_{n}$ with respect to the central line: $\Delta w_{n}=\Delta w_{0}+\Delta w_{\text {jitter }}\left(N-N_{0}\right)^{2}$ where $N$ is a generic mode number, $N_{0}$ is the mode number corresponding to the minimum linewidth, and $\Delta w_{0}$ accounts for other phase noise contributions ${ }^{7,32}$. As the resolution of our RDSHI measurements was $<10 \mathrm{kHz}$, we estimate a pulse-jitter bandwidth $\Delta w_{\text {jitter }}<10 \mathrm{kHz}$.

\section{References}

1. Keller, U. Recent developments in compact ultrafast lasers. Nature 42, 831-838 (2003).

2. Haus, H. Mode-locking of lasers. IEEE J. Sel. Top. Quant. 6, 1173-1185 (2000).

3. Akhmediev, N. N., Ankiewicz, A. \& Soto-Crespo, J. Multisoliton solutions of the complex Ginzburg-Landau equation. Phys. Rev. Lett. 79, 4047-4051 (1997).

4. Bulent, O. et al. Soliton-similariton fibre laser. Nature Photon. 4, 307-311 (2010).

5. Essiambre, R. J., Kramer, G. \& Winzer, P. J. Capacity limits of optical fibre networks. J. Lightw. Technol. 28, 662-701 (2010).

6. Cotter, D. et al. Nonlinear optics for high-speed digital information processing Science 286, 1523-1528 (1999).

7. Habruseva, T. et al. Optical linewidth of a passively mode-locked semiconductor laser. Opt. Lett. 34, 3307-3309 (2009).

8. Jiao, Z. J. et al. C-band InAs/InP quantum dot semiconductor mode-locked laser emitting 403-GHz repetition rate pulses. IEEE Photon. Technol. Lett. 23, 543-545 (2011).

9. Franco, P. et al. Self-induced modulational-instability laser. Opt. Lett. 20, 2009-2011 (1995)

10. Yoshida, E. \& Nakazawa, M. Low-threshold 115-GHz continuous-wave modulational-instability erbium-doped fibre laser. Opt. Lett. 22, 1409-1411 (1997).

11. Quiroga-Teixeiro, M. et al. Passive mode locking by dissipative four-wave mixing. J. Opt. Soc. Am. B 15, 1315-1321 (1998).
12. Sylvestre, T. et al. Self-induced modulational instability laser revisited: normal dispersion and dark-pulse train generation. Opt. Lett. 27, 482-484 (2002).

13. Schröder, J., Vo, T. D. \& Eggleton, B. Repetition-rate-selective, wavelengthtunable mode-locked laser at up to $640 \mathrm{GHz}$. Opt. Lett. 34, 3902-3904 (2009).

14. Schröder, J. et al. Dynamics of an ultrahigh-repetition-rate passively modelocked Raman fibre laser. J. Opt. Soc. Am. B 25, 1178-1186 (2008).

15. Zhang, S. et al. Passive mode locking at harmonics of the free spectral range of the intracavity filter in a fibre ring laser. Opt. Lett. 30, 2852-2854 (2005).

16. Kippenberg, T. J., Holzwarth, R. \& Diddams, S. A. Microresonator-based optical frequency combs. Science 332, 555-559 (2011).

17. Del'Haye, P., Arcizet, O., Schliesser, A., Holzwarth, R. \& Kippenberg, T. J. Full stabilization of a microresonator-based optical frequency comb. Phys. Rev. Lett. 101, 053903 (2008).

18. Del'Haye, P. et al. Optical frequency comb generation from a monolithic microresonator. Nature 450, 1214-1217 (2007).

19. Grudinin, I. S., Yu, N. \& Maleki, L. Generation of optical frequency combs with a $\mathrm{CaF}_{2}$ resonator. Opt. Lett. 34, 878-880 (2009).

20. Levy, J. S. et al. CMOS-compatible multiple-wavelength oscillator for on-chip optical interconnects. Nature Photon. 4, 37-40 (2010).

21. Razzari, L. et al. CMOS-compatible integrated optical hyper-parametric oscillator. Nature Photon. 4, 41-45 (2010).

22. Ferdous, F. et al. Spectral line-by-line pulse shaping of on-chip microresonator frequency combs. Nature Photon. 5, 770-776 (2011).

23. Schawlow, A. L. \& Townes, C. H. Infrared and optical masers. Phys. Rev. 112, 1940-1949 (1958).

24. Yoshida, M., Ono, A. \& Nakazawa, M. $10 \mathrm{GHz}$ regeneratively modelocked semiconductor optical amplifier fibre ring laser and its linewidth characteristics. Opt. Lett. 32, 3513-3515 (2007).

25. Carmon, T., Yang, L. \& Vahala, K. Dynamical thermal behavior and thermal self-stability of microcavities. Opt. Express 12, 4742-4750 (2004).

26. Ferrera, M. et al. Low power four wave mixing in an integrated, micro-ring resonator with Q=1.2 million. Opt. Express 17, 14098-14103 (2009).

27. Ferrera, M. et al. Low-power continuous-wave nonlinear optics in doped silica glass integrated waveguide structures. Nature Photon. 2, 737-740 (2008).

28. Pasquazi, A. et al. Sub-picosecond phase-sensitive optical pulse characterization on a chip. Nature Photon. 5, 618-623 (2011).

29. Haboucha, A., Leblond, H., Salhi, M., Komarov, A. \& Sanchez, F. Analysis of soliton pattern formation in passively mode-locked fibre lasers. Phys. Rev. A 78, 043806 (2008).

30. Dawson, J. W., Park, N. \& Vahala, K. J. An improved delayed self-heterodyne interferometer for linewidth measurements. IEEE Photon. Technol. Lett. 4, 1063-1066 (1992).

31. Linde, D. Characterization of the noise in continuously operating mode-locked lasers. Appl. Phys. B 39, 201-217 (1986).

32. Haus, H. A. \& Mecozzi, A. Noise of mode-locked lasers. IEEE J. Quantum Electron. 29, 983-996 (1993).

\section{Acknowledgements}

This work was supported by the Canadian FQRNT and NSERC, and the Australian Research Council (ARC) Centres of Excellence and Discovery Projects programs. M.P. acknowledges the support of the Marie Curie People project TOBIAS PIOFGA-2008-221262. We also acknowledge support from QPS Photonics, Enablance and MPB Communications. Finally, we thank Stefano Trillo for the discussions on the theoretical basis of nonlinear dissipative systems and Tudor Johnston for a critical revision of the manuscript.

\section{Author contributions}

M.P. and A.P. designed the laser operating scheme and principle of operation. M.P. and A.P. designed the short-length amplifier used in the stable laser design. M.P., A.P. and Y.P. built the laser prototype used in the paper as a proof of concept. A.P. developed the theoretical and numerical analyses. S.T.C. and B.E.L. designed and fabricated the resonator exploited in the laser design. D.J.M. and R.M. supervised the project. M.P., A.P., D.M. and R.M. wrote the manuscript.

\section{Additional information}

Competing financial interests: The authors declare no competing financial interests.

Reprints and permission information is available online at http://npg.nature.com/ reprintsandpermissions/

How to cite this article: Peccianti, M. et al. Demonstration of a stable ultrafast laser based on a nonlinear microcavity. Nat. Commun. 3:765 doi: 10.1038/ncomms1762 (2012)

License: This work is licensed under a Creative Commons Attribution-NonCommercialShare Alike 3.0 Unported License. To view a copy of this license, visit http:// creativecommons.org/licenses/by-nc-sa/3.0/ 